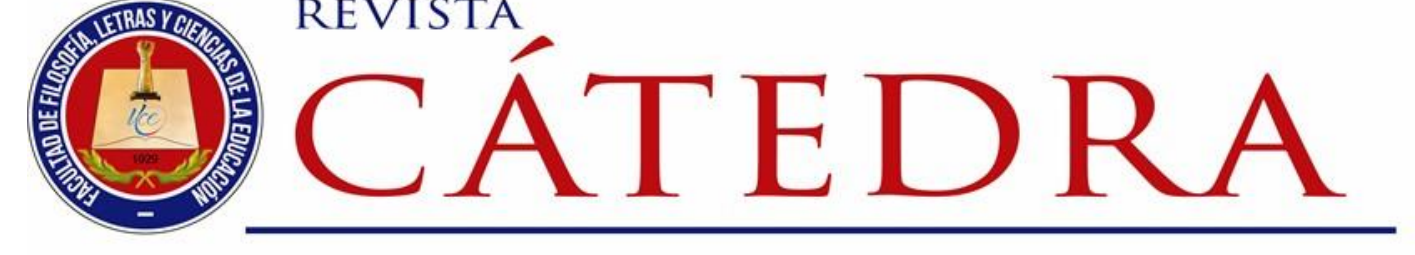

\title{
Redes sociales en universidades del país. Análisis descriptivo y planteamiento de una posible solución para mejorar el impacto en la actividad académica
}

Social networks in universities of the country: Descriptive analysis and approach to a possible solution to improve the impact on academic activity

Esteban Gordon-Salcedo

Universidad Central del Ecuador, Quito, Ecuador edgordon@uce.edu.ec https://orcid.org/0000-0001-9930-8213

Frans Noguera-Vásconez

Universidad Central del Ecuador, Quito, Ecuador dnoguera@uce.edu.ec https://orcid.org/0000-0002-8308-4814

Mario Morales-Morales

Universidad Central del Ecuador, Quito, Ecuador mmoralesm@uce.edu.ec https://orcid.org/0000-0002-7493-8072

Santiago Morales-Cardoso

Universidad Central del Ecuador, Quito, Ecuador smorales@uce.edu.ec http://orcid.org/0000-0002-3833-9654,

(Recibido: 11/07/2019; Aceptado: 8/08/2019; Versión final recibida: 5/09/2019)

Cita del artículo: Gordón-Salcedo, E., Noguera-Vasconez, F., Morales-Morales, M., y MoralesCardoso, S. (2019). Redes sociales en universidades del país. Análisis descriptivo y planteamiento de una posible solución para mejorar el impacto en la actividad académica. Revista Cátedra, 2(3), 16-38.

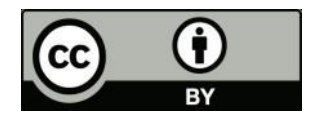




\section{Resumen}

El uso actual que dan los estudiantes universitarios a las redes sociales, y la postura de los centros educativos al momento de incorporar estos medios a sus planes académicos, es el objetivo y fundamento que justifica este trabajo para que se analice el uso de la data que proviene de publicaciones extraídas de FanPages de tres universidades de Quito, además de los comentarios y reacciones de los usuarios a dichas publicaciones. La comparación entre el uso para lo académico y para el ocio es el principal eje que se examinó, mostrando sus resultados a través de gráficos y estadísticas. En base en este análisis se plantea un posible recurso basado en la tecnología de los chatbots para generar un mayor compromiso y fidelidad por parte de los universitarios hacia su centro de educación.

De la investigación realizada se pudo comprobar que los estudiantes buscan en las redes sociales generar experiencias y sentimientos, por lo cual interactúan más con las páginas de ocio que con las académicas. Ahí encuentran una cantidad de palabras y publicaciones que les permite identificarse y convertirse en un seguidor fiel, que tiene como característica principal, el compartir, comentar y reaccionar con la mayoría de los posteos.

\section{Palabras clave}

Academia, canales conversacionales, compromiso, ocio, redes sociales.

\section{Abstract}

This manuscript aimed to examine the use made by university students to social networks and the position held by educational centers when incorporating such means to their academic plan, being the latter the objective of this study in order to analyze the data based on publications taken from FanPages of three universities in Quito, in addition to comments and reactions by users of such releases. Comparison between the use for academic purposes and for leisure is the main parameters considered, and results were shown trough graphics and statistics. Based on such analysis, a possible resource was proposed, based on chatbots technology in order to generate more commitment and loyalty by university students to educational establishment.

It was found that students use social networks to generate experiences and feelings, for which an interaction is generated mostly with leisure websites in comparison to academic ones, where a great amount of words and publications are found, allowing to identify themselves and become a follower, whose main characteristics is share, comment and react with most of the postings.

\section{Keywords}

Social networks, engagement, academy, leisure, conversational channels.

\section{Introducción}

En este trabajo investigativo se plantearán otros tipos de obtención de datos tanto cualitativos como cuantitativos, obligando así a un distinto tipo de análisis de los mismos para la obtención de información inexplorada aún. Para esto se hará uso de las nuevas tecnologías que han surgido para el análisis de información no estructurada, con el propósito de medir el comportamiento e impacto en los estudiantes y plantear una posible solución que promueva el conocimiento académico usando la red social Facebook. Esto supondrá una gran ventaja por parte de los centros educativos para impactar de una mejor 
manera en los estudiantes y aprovechar al máximo las funciones de las redes sociales en auge por parte de los universitarios.

Tomando como premisa el estado actual del conocimiento, se ha planteado la hipótesis de que el engagement (compromiso) en las FanPages académicas por parte de los estudiantes universitarios de la ciudad de Quito, no supera al tercio del engagement generado por páginas dedicadas al ocio y entretenimiento.

El objetivo es realizar un estudio de los comportamientos y expectativas del uso de las redes sociales por parte de los estudiantes de tres universidades de renombre en la ciudad de Quito. Los datos se recolectarán de las FanPages de las universidades que hayan sido seleccionadas y sus pares dedicadas al ocio, estos datos nos permitirán observar qué utilidad le dan las autoridades y estudiantes a esta red. La comparación entre el uso académico y para el ocio será el principal eje en este trabajo, el cual se presentará en resultados estadísticos, y soluciones que aporten a las entidades educativas universitarias. Las limitaciones que tendrá el trabajo son que los datos extraídos para el posterior análisis serán solamente de la red social Facebook. Los datos para examinar serán de los últimos tres meses.

El diseño para este trabajo es del tipo descriptivo. Su objetivo es la identificación de causas y evaluación de sus efectos por lo cual permitirá analizar las variables planteadas, controlar y medir cualquier cambio de una manera eficiente y ordenada. Este estudio resume los elementos y consideraciones más importantes que fueron desarrollados en su totalidad en el trabajo de tesis de (Gordon-Salcedo y Noguera-Vásconez, 2018).

El presente documento está estructurado como sigue; la introducción contiene la fundamentación teórica; en la metodología se describe cada paso efectuado en la investigación; la discusión de resultados presenta un análisis de las mediciones encontradas tanto desde el punto de vista por escenario como por consulta; finalmente el capítulo de conclusiones resume los hallazgos, así como posibles líneas futuras de la investigación.

\subsection{Marco teórico}

\subsubsection{Conceptos Generales}

- Redes sociales digitales.

Entre algunos autores se define a una red social digital como un entorno en la web, cuyo objetivo es facilitar a las personas el establecimiento de relaciones entre sí, crear comunicaciones, organizar comunidades, y formar sitios con distintos contenidos. Con el objetivo principal de compartir información desde cualquier parte del mundo, siendo cualquier persona un potencial creador de información (Bustos, Flores, y Flores, 2016). Los jóvenes presentan gran conocimiento en las redes sociales y una buena disposición de usarlas con fines didácticos, de esta manera al mismo tiempo que interactúan y se divierten en ellas, van generando un valor académico consolidando formas de estudio cooperativo óptimas (Espuny et al., 2011). En el estudio realizado por (Gómez et al., 2012) se señalan los principales motivos para el uso de redes sociales, y éstos son: entorno general (amigos, familiares) un $75 \%$, entretenimiento un $61,8 \%$ y estudios un $24,7 \%$.

- Influencia de las redes sociales en el campoacadémico.

A pesar del gran potencial que muestran las redes sociales digitales, éstas se pueden convertir en una gran amenaza si no se les da el uso debido, ya que pueden llegar a ser un distractor para los estudiantes y jóvenes en general, en quienes el promedio de uso para actividades dedicadas al entretenimiento es de 7 horas, llegando en muchos casos a un tiempo de hasta 14 horas (Bustos et al., 2016). El análisis llevado 
a cabo por Huang (2018) apoya estos argumentos, donde se calculó la correlación entre el uso de las redes sociales digitales y el rendimiento académico de los estudiantes, y se halló una correlación negativa muy pequeña. Todos estos factores obligatoriamente les restan tiempo de estudio a los alumnos, afectando directamente al rendimiento académico, por estos motivos muchas universidades se han mostrado reacias al uso de las redes sociales en los entornos educativos, alegando que en ellos se fomenta el ocio y que las publicaciones carecen de un buen sustento científico (Rodríguez y Patricia, 2012). Sin embargo, las redes sociales poseen un gran potencial para mejorar actividades de carácter cognitivo y educativo, valiéndose de una correcta supervisión por parte de los docentes (Bustos et al., 2016).

- Análisis de las redes sociales.

Las redes sociales ofrecen la capacidad de encontrar patrones y propiedades en las interacciones de los usuarios, para ser estudiados con el objetivo de entender la relación y el comportamiento entre los actores involucrados mediante la aplicación de algunas técnicas como uso de redes y teorías gráficas (Dakiche et al., 2018). Una técnica para estos estudios es identificando la forma en que se establecen comunidades, agrupaciones o clústeres, es decir, una alta densidad de interacciones entre individuos del mismo grupo y una baja densidad de interacciones entre individuos de distintos grupos (Leskovec et al., 2008). Otra técnica es el análisis de contenido, cuya característica es dar información acerca de datos no estructurados y la fluidez en el texto semántico de las publicaciones (Antolín-Prieto, 2012), ayudando a establecer características predominantes de los mensajes para enmarcar a las publicaciones en diferentes temas (Onieva, 2017).

\section{- Engagement.}

El término anglosajón engagement ha ido evolucionando a través del tiempo, siendo una medida de la subvención que existe entre los clientes y la empresa (Erat, Desouza, Schäfer-Jugel y Kurzawa, 2006). Si bien existe gran variedad de conceptualizaciones de esta medida, muchos investigadores coinciden en que el engagement es un estado mental, que lleva a un sujeto a focalizarse en una marca, un medio o un servicio y realizar interacciones frecuentes y continuas con el objeto focal, las cuales van más allá de realizar una compra o una transacción (Thakur, 2018). Su medición se obtiene observando el grado de participación que tienen los usuarios hacia la marca o servicio, ya sea consumiéndolo, compartiéndolo, publicándolo, etc. (Wagner y Majchrzak, 2007). Este compromiso se basa en un collage de experiencias, que son las creencias que tiene un sujeto de cómo puede adaptar una página web con su vida (Calder et al., 2009). El alto nivel de satisfacción generada por estas experiencias conduce a la lealtad de la persona, la cual se manifiesta en forma de readquisición, en forma de apoyo o en forma de propaganda boca a boca (Anaza y Zhao, 2013). Otro factor asociado al compromiso del cliente es la confianza en la empresa (Kim et al., 2009), que conduce a la participación del usuario no solo para realizar una adquisición inmediata, sino para mantenerse en contacto con la marca. Según (Achen, 2016) la construcción del Engagement es importante para obtener información del intercambio de productos y servicios de determinada empresa.

- FanPages.

Son páginas que permiten a los usuarios interactuar y afiliarse con una marca, de la misma manera en que interactúan con otros perfiles de usuario privados (Perez et al., 2018). Así las personas tienen un canal para seguir a sus marcas favoritas, con la opción de compartir y recomendar a sus amigos sus intereses, ya que cuando un cliente le marca con me gusta a una de estas páginas, ya se convierte en un fan de 
ella, y esta información se hace visible en el perfil del usuario. Las FanPages tienen una apariencia de páginas individuales de usuario, pero poseen la ventaja única de enviar mensajes rápidamente a una gran cantidad de seguidores, por esta razón muchas empresas los utilizan como una herramienta de comunicación empresarial para constituir diálogos continuos con sus clientes (Kudeshia et al., 2016), los cuales están siendo ampliamente aceptados, consiguiendo un aumento de ventas significativo para las organizaciones (Pöyry et al., 2013).

\subsection{Redes sociales y sus aplicaciones}

\section{- Educación.}

La nueva tendencia a nivel mundial para una entrega educativa fue denominada MOOC (Massive Open Online Courses), que se basa en interacciones de colaboración entre todos los usuarios mientras se desarrollan habilidades digitales, con acceso libre a toda la información y recursos que se necesiten, sin requerir el asistir a un salón de clases, con la ventaja de que los estudiantes avanzarán a su propio ritmo y en los tiempos más apropiados para cada uno. En lo económico, estos cursos reducen los gastos en la enseñanza al combinar servicios de tutoría con el aprendizaje en línea y redes sociales gratuitas. Todo esto hace que esta nueva forma de educación sea muy atractiva para estudiantes de todo el mundo. Algunos proveedores de MOOC son: Coursera, edX, Udacity, FUN, Iversity, FutureLearn, Rwaq, Edraak, entre otros (Brahimi y Sarirete, 2015).

- Marketing.

Hoy en día las redes sociales también se han posicionado como ambientes idóneos para dar a conocer una marca, un servicio o un producto. Haciendo uso de las distintas funcionalidades que ofrecen, como por ejemplo: la capacidad de subir información, el poder compartir y publicar contenido escrito o archivos multimedia, la facilidad que brindan para crear redes de personas alrededor de todo el mundo, el permitir la personalización de páginas comerciales para crear una representación de su propia identidad (Gonzales y Hancock, 2010), y la capacidad que brindan para dar un seguimiento a las reacciones de los usuarios con un producto, acrecentando así el campo de visión que se tiene de un negocio para poder tomar decisiones con base a los resultados obtenidos (Herrera-Torres et al., 2017).

- Inteligencia artificial en las redes sociales.

Uno de los aspectos más importantes en las redes sociales es el establecimiento de canales conversacionales, interacciones que no sea solamente unidireccionales, sino bidireccionales, esto desarrolla en el usuario un sentimiento positivo hacia el servicio, marca o página (Onieva, 2017). Para lograr crear estos canales de comunicación se puede hacer uso de las nuevas tecnologías, como los agentes conversacionales virtuales basados en inteligencia artificial, los cuales permiten crear ambientes universitarios propicios para la comunicación entre profesores, alumnos y personal. Aún hoy en día no se explota todo el potencial que puede brindar esa tecnología, pero ya se han llevado a cabo algunos casos de éxito, como la herramienta Agent SocialMetric, que le ayuda al docente a estar al tanto del estado actual del aula a su cargo (Kuz et al., 2015).

Para el desarrollo de esta herramienta se valieron de un agente de software conversacional inteligente o chatbot. Un chatbot es un software que usa el lenguaje natural para interactuar con los usuarios, simulando así un comportamiento humano que se basa en modelos y patrones de diálogo de las personas, para actuar como mediadores en la distribución de tareas cognitivas entre las máquinas y las personas. Han existido varios términos para referirse a estos programas, como: agente virtual, chatterbot, sistema de diálogo, entre 
otros. Hoy en día se han desarrollado una amplia gama de chabots para muchos servicios y su uso sigue en un aumento exponencial (Ciechanowski et al., 2018).

El agente de software de interfaz conversacional que se usó en esta herramienta fue uno denominado Albert, quien actúa como mediador en la relación que se da entre el profesor y sus alumnos. Así la herramienta Agent socialMetric logra distinguir las preguntas de los usuarios y luego elabora la respuesta adecuada a la oración recibida, creando líneas de colaboración y un excelente ambiente para el desarrollo de trabajos pedagógicos. Una característica importante de esta herramienta es la posibilidad de que el docente puede conversar con Albert, para extraer información acerca del clima social de su aula, encontrando así perfiles de alumnos como: los molestosos, los líderes, los amigos, entre otros, de esta manera se puede contribuir para desarrollar un ambiente cómodo para el aprendizaje. Para estudios futuros se pretende ampliar los métodos de comunicación con el agente conversacional, con canales aptos para personas con alguna discapacidad visual, implementando funciones de audio al chatbot (Kuz et al., 2015).

\subsection{Estudio de casos}

En la actualidad se han llevado varios análisis del uso de las redes sociales en diferentes campos, como son: el deporte, la academia, el comercio etc. A continuación, se detallan algunos de estos casos que marcan varios ejemplos para estudios posteriores.

\subsubsection{Compromiso digital e identidad social de los fans deportivos}

El caso de los Equipos de la Premier League en Facebook (Herrera et al., 2018). El objetivo del estudio fue analizar el nivel de fanatismo por parte de los seguidores a las principales Fanpages de los equipos de la Premier League, a través de la correlación existente entre las interacciones y las publicaciones de la página. Las interacciones de los usuarios que se tomaron en cuenta fueron: me gusta, comentar y compartir.

Estos tres tipos de reacciones, según el estudio, indican un distinto grado de compromiso, por ejemplo, al responder a una publicación con una acción Me gusta.

\subsubsection{Uso de las redes sociales por las universidades públicas andaluzas. Análisis de contenido de la cuenta oficial de Twitter (Onieva, 2017)}

En este estudio se analizó cómo las universidades andaluzas usaban las redes sociales para llegar al público. Se establecieron dos tipos de públicos: interno (profesores, alumnos, administrativos y de servicios) y un público exterior (otras empresas, exalumnos, alumnos buscando ingresar a la universidad y público en general). Para reducir el campo de estudio se analizó solamente las cuentas oficiales de las universidades en Twitter, en los meses de febrero de los años 2014, 2015 y 2016. Similar al estudio realizado en los equipos de fútbol, en este estudio se identificaron en cada mensaje de Twitter las interacciones por parte de los usuarios, que serían las acciones de: me gusta, que indica un interés básico por el mensaje, o retuitear, lo que indica el deseo de dar a conocer el mensaje a otras personas.

El análisis de contenido fue un aspecto importante para establecer cuáles son las características de los mensajes que predominan en las redes sociales por parte de las universidades de la región andaluza. Se pudo observar que la temática más usada fue con respecto a cursos, información de conferencias, jornadas y casas abiertas, y los temas menos abarcados fueron acerca de empleo, información para los alumnos, obras sociales y voluntarias. Confirmando así el problema comunicativo mencionado por (Paniagua et al., 2012) que las universidades han adoptado al Internet y a las redes sociales como un medio de información y no han enfatizado en la comunicación con sus alumnos, profesores y personal administrativo. En este estudio también se aconseja que las universidades deberían considerar la idiosincrasia de cada red social para establecer temáticas propias

Licencia Creative Commons Atribución 4.0 Internacional (CC BY 4.0)

Revista Cátedra, 2(3), pp. 16-38, septiembre-diciembre 2019. e-ISSN: 2631-2875

https://doi.org/10.29166/catedra.v2i3.1713 
para cada una de éstas, con el fin de captar de una mejor manera la atención y el compromiso de los clientes internos y externos, y publicar contenido atractivo para el usuario de cada red y no enviar mensajes solo por llenarse de publicaciones, lo cual podría incluso ser perjudicial para la página como afirma (Onieva, 2014).

\subsubsection{Incidencia de las redes sociales en el rendimiento académico de los estudiantes de la universidad de la Guajira (Colombia) (Martelo et al., 2017)}

En este estudio se pretende encontrar la influencia de las redes sociales digitales en el rendimiento académico de los estudiantes universitarios. La metodología que se usó es de tipo no experimental transversal descriptiva, en una muestra de 368 alumnos del primer semestre de la universidad de La Guajira en Colombia, en el año 2016. El análisis se basó en una prueba de hipótesis con diferencia de proporciones muestrales, comparando y obteniendo la variación de las calificaciones entre dos periodos de tiempo; uno al hacer uso de las redes sociales, y el otro sin hacer uso de estos sitios web. Como resultados se observa que existe gran influencia negativa de las redes sociales en el rendimiento académico de los alumnos, resultados que corroboran los estudios de (Junco, 2012). También se pudo apreciar que la red social de mayor uso es Facebook, seguida de Twitter, Instagram y YouTube.

\section{Metodología}

La presente investigación es de tipo aplicada, con un diseño descriptivo simple, de corte longitudinal retrospectivo, por tanto, el diseño a ser utilizado relaciona el tiempo, la acción y la audiencia, dichas dimensiones nos ayudan a conceptualizar el proceso de investigación estudio-impacto (Willet, 1974). En virtud de observar y medir la incidencia del uso y manejo de las FanPages de las universidades a estudiar, así como de las páginas dedicadas al ocio denominadas MEMES, correspondientes a cada centro universitario. Los pasos a seguir son:

- Selección y características de la muestra

Determinar la muestra idónea de publicaciones a examinar, que nos permitan encontrar tendencias, comportamientos, características e interacciones que realizan los estudiantes de las tres universidades más transcendentes del país.

- Definición y características de variables

En base al estado actual del conocimiento y a la hipótesis planteada, determinar las variables e interacciones necesarias para el análisis descriptivo de esta investigación.

- Elección y uso de la herramienta de apoyo

Comparar herramientas de análisis y Benchmarking de redes sociales que faciliten la extracción y análisis de datos.

- Análisis y reconocimiento de patrones de la data

En esta etapa de la investigación, se debe realizar comparación de las variables estadísticas de las FanPages a través de filtros, gráficos, tablas, que permitan determinar rasgos comunes que buscan los estudiantes universitarios para interactuar y convertirse en Fans.

- Posible solución para un mejor impacto en el área académica.

Finalmente, ya determinados los rasgos y comportamientos que permiten realizar una mayor interacción entre los estudiantes y las FanPages, desarrollar un prototipo de software que mejore y posicione las páginas educativas. 


\section{Cálculos y resultados}

\subsection{Selección de la muestra}

El grupo de estudio al que se le aplicó el seguimiento fue de los estudiantes pertenecientes a tres universidades con gran trascendencia en la ciudad de Quito entre públicas y privadas: La Universidad Central del Ecuador por ser la universidad con más historia en el Ecuador, la Escuela Politécnica Nacional al ser un referente de alto nivel académico en el país, y la Pontificia Universidad Católica del Ecuador por tener el puesto 71 en el ranking de las universidades Latinoamericanas en QS Latin American University Ranking 2018. El promedio total de personas del conglomerado que interactuarían con todas las FanPages a comparar, es alrededor de los ochenta mil usuarios entre hombres y mujeres, como se ve en el cuadro 1.

\section{Género}

\begin{tabular}{lrrr}
\hline Nombre & Estudiantes & F & M \\
\hline Universidad Central del Ecuador & 65.259 & 38.133 & 27.126 \\
Escuela Politécnica Nacional del Ecuador & 9.114 & 2.734 & 6.380 \\
Pontificia Universidad Católica del Ecuador & 14.445 & 8.667 & 5.778
\end{tabular}

Cuadro 1. Cuadro comparativo de las universidades y el número de estudiantes

Para la selección de la red social a estudiar se ha tomado en cuenta el porcentaje de uso de éstas por parte de los estudiantes universitarios como se puede ver en la Figura 1, la red que se usó para la investigación es la red social Facebook. Al encontrarse en un rango de aceptación muy elevado entre los universitarios y por las ventajas que presenta al momento de extracción y clasificación de los datos.

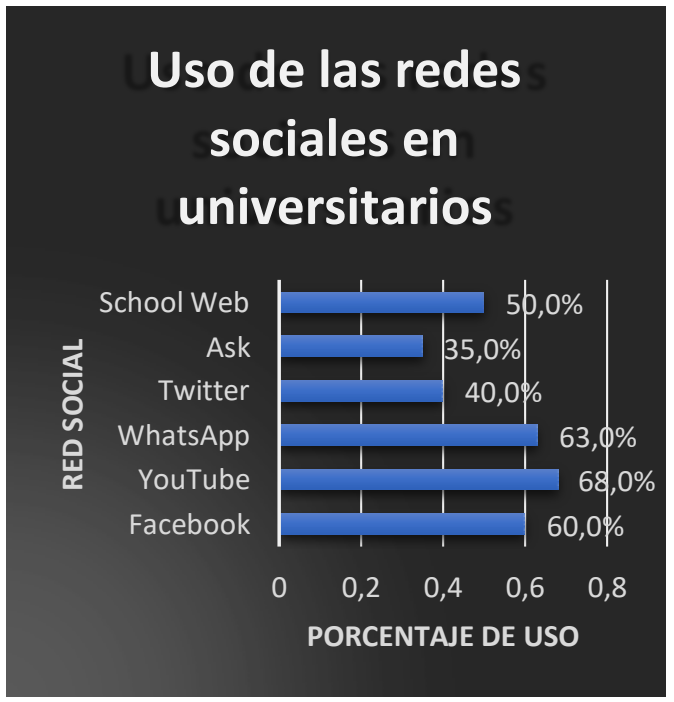

Figura 1. Porcentaje de uso de las redes sociales por estudiantes universitarios (Brahimi y Sarirete, 2015). 


\subsection{Definición y características de variables}

Para la investigación se han establecido variables que permitan corroborar las estadísticas de la hipótesis, se ha planteado en base a los estudios de casos presentados anteriormente en la metodología, con guía en los comportamientos y tendencia de los jóvenes universitarios al momento de interactuar en las redes sociales (Facebook), conglomerándolas en 3 grupos, presentados así:

\section{- Variables independientes}

En este grupo se han establecido aquellas variables que permiten establecer una relación directa entre la publicación y la opinión de los estudiantes.

- Interacciones digitales

Son las acciones que se puede realizar en una publicación, comentario, link, o video en Facebook, donde el número de ellas determina que tan fuerte ha sido su engagement con la comunidad, determinando si fue exitosa, o simplemente pasó desapercibida en la navegación. Las interacciones, se las ha clasificado de forma jerárquica, como indica la Figura 2, siendo la más apetecida por los administradores de las FanPages la opción de compartir, seguida de comentarios y reacciones.

\section{- Variables dependientes.}

Aquí se han determinado los dos principales ejes de la investigación, siendo la unión de ellos, el universo de estudio para determinar el comportamiento y uso de los jóvenes universitarios en Facebook, cada uno siendo dependientes de las interacciones que se ven sometidos a las publicaciones realizados por sus administradores. Los grupos son:

- Académico.

Aquí se engloba las FanPages oficiales de cada una de las universidades a analizar, donde su objetivo es el de informar y rescatar noticias notables de sus instituciones, queriendo llegar a mejorar su imagen en el ámbito social.

- Ocio.

Aquí se enfatiza en las FanPages que realizan una crítica social, mediante memes y contenido generalmente humorista, donde su objetivo es criticar las características que definen a las demás universidades, como los problemas que también tienen las mismas, siendo muy volátiles en el tiempo.

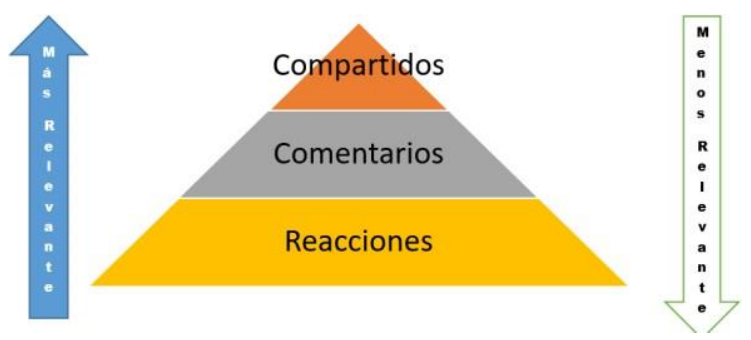

Figura 2. Relevancia de las interacciones

\section{- Variables intervinientes}

Se ha definido aquí, a todas las variables que pueden interferir en los resultados, iniciando con el elemento que caracteriza a los jóvenes universitarios, siendo una variable susceptible en el tiempo, denominada Emoción. Lo emocional como elemento que se enfatiza en los jóvenes universitarios, hace que se sientan identificados con las opciones de interacción digital, por tanto, cabe la reflexión en 
hablar de fan o seguidores. Los fans de los que le dan me gusta a todo lo que publica una marca, casi sin mayor conocimiento del contenido de lo publicado, pues una vez que ha ganado sus emociones, prima la interacción digital hasta impulsiva; mientras que los seguidores se mantienen observando cada publicación en espera de que a su criterio le parezca importante, ahora, probablemente ser seguidor sea la antesala a convertirse en un fan.

Determinamos que el rango de edad de un estudiante universitario varía entre los 18 a 35 años y se los ha clasificado en tres subcategorías que son:

○ Período inicial.

Aquellos que cursan un nivel propedéutico, donde se desea tener una mejor imagen, y adaptabilidad con el grupo, dejándose influenciar grandemente por las tendencias y opiniones del conjunto.

- Período medio.

Aquellos estudiantes que se encuentran en los niveles medios, teniendo un criterio mejor formado de todo lo que se ha venido presentando en su carrera universitaria, defendiendo y apoyando a su casa de estudio en cada una de las publicaciones.

- Período superior.

Aquellos estudiantes, próximos a graduarse, con características formadas, donde su enfoque ya no depende de las opiniones generales, donde su intención es informarse de aquello que pueda construir mejor su carrera profesional y educativa.

\subsection{Elección y uso de la herramienta de apoyo}

El elegir una herramienta óptima para el estudio a realizarse es de vital importancia, ya que los datos que se obtengan a partir de la misma, serán la base de todo el análisis. Se compararon varias herramientas para elegir la más adecuada para el estudio a realizar y se determinó hacer uso de la herramienta de Business Intelligence (BI) para redes sociales Social Scan de la compañía Golden Comunicaciones.

Se la ha seleccionado por la facilidad que ofrece al momento de reconocer y analizar las métricas de las redes sociales presentes en las páginas de Facebook de alguna empresa y de su competencia. La información se la puede presentar en una interfaz intuitiva y dinámica, ya que esta herramienta convierte automáticamente los datos que se han extraído a gráficos estadísticos para su mejor entendimiento. Además, que cuenta con las funciones de líneas de tiempo, y de filtración de datos drill-down y roll-up que brindan una gran ayuda para el manejo de la información aumentando así la comprensión de los resultados.

Social Scan permite reconocer y analizar la polaridad en los comentarios de los usuarios y realizar un análisis de contenido a todas las publicaciones que se requieran de una FanPage, lo cual será muy positivo a la hora de analizar los comportamientos de los usuarios y de las universidades en la red social. La herramienta además brinda gran destreza para la extracción de datos en tiempo real a una gran velocidad, ahorrando tiempo en la detección, extracción y visualización de la data. Y a diferencia de otras herramientas, cuenta con soporte local en Ecuador.

\subsection{Análisis y reconocimiento de patrones en la data}

Con la definición de todas las variables, y con la ayuda de la herramienta elegida, se ha determinado construir un tablero personalizado, donde se pueda enfocar en cada uno de los temas a analizar, enfatizando y determinando cuáles son las opiniones, interacciones y reacciones que motivan a tener un mejor engagement de nuestros principales ejes que son el Ocio y el Académico.

Licencia Creative Commons Atribución 4.0 Internacional (CC BY 4.0)

Revista Cátedra, 2(3), pp. 16-38, septiembre-diciembre 2019. e-ISSN: 2631-2875

https://doi.org/10.29166/catedra.v2i3.1713 
- Posicionamiento General

Debido al estudio de la muestra anteriormente mencionada se ha plasmado la ubicación de las FanPages a estudiar, observando en la Figura 3 cuál de ellas genera una mayor interacción en el ámbito social.

En el cuadrante de calificación se ha observado que la FanPage Memes UCE Quito, tiene el mayor número, acercándose a las 60.000 reacciones, superando además los 2.000 comentarios, quedando sus compañeras de grupo Memes PUCE y Poli Memes en las primeras jerarquías de posicionamiento, siendo relegadas las demás FanPages oficiales, con la observación de que PUCE_Ecuador se acerca al grupo de OCIO.

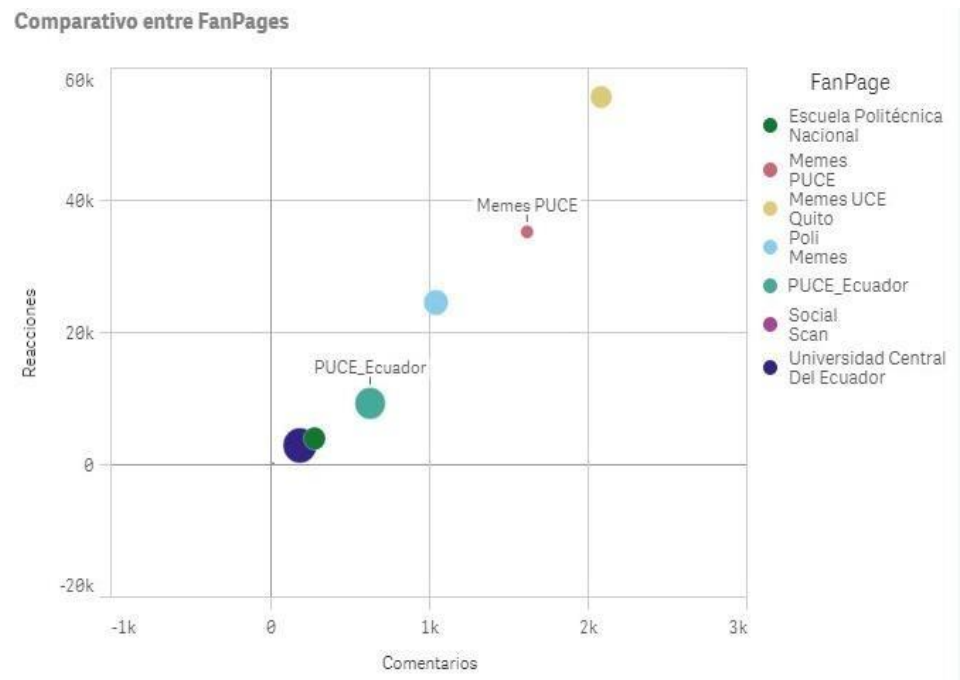

Figura 3. Posicionamiento FanPages reacciones vs comentarios

Con base en estos datos se construyó una tabla que muestra las posiciones de las FanPages, por interacciones con los estudiantes estableciéndose las posiciones como se muestra en el cuadro 2.

\begin{tabular}{|c|c|c|c|c|c|c|}
\hline FanPage & $\begin{array}{l}\mathrm{N}^{\circ} \text { de } \\
\text { Seguidores }\end{array}$ & $\begin{array}{l}\mathrm{N}^{\circ} \text { de } \\
\text { Publicaciones }\end{array}$ & $\begin{array}{l}\mathrm{N}^{\circ} \\
\text { Compartidos }\end{array}$ & $\begin{array}{l}\mathrm{N}^{\circ} \\
\text { Comentarios }\end{array}$ & $\begin{array}{l}\mathrm{N}^{\circ} \\
\text { Reacciones }\end{array}$ & $\begin{array}{l}\mathrm{N}^{\circ} \text { de } \\
\text { Interacciones }\end{array}$ \\
\hline Memes UCE & 40.274 & 140 & 10.540 & 2.079 & 55.745 & 68.364 \\
\hline $\begin{array}{l}\text { Memes } \\
\text { PUCE }\end{array}$ & 14.427 & 140 & 28.368 & 1.613 & 35.355 & 65.336 \\
\hline Poli Memes & 52.574 & 140 & 3.204 & 1.040 & 24.665 & 28.909 \\
\hline PUCE Ec & 81.495 & 140 & 1.531 & 626 & 9.411 & 11.568 \\
\hline EPN & 41.593 & 140 & 938 & 276 & 4.093 & 5.307 \\
\hline UCE & 100.31 & 140 & 697 & 184 & 3.039 & 3.920 \\
\hline
\end{tabular}

Cuadro 2. Posicionamiento de las Fanpages ordenadas por interacciones

\section{- Promedio de publicaciones diarias}

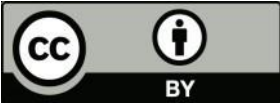


Para poder efectuar un análisis más detallado fue necesario crear nuevas métricas, entre las cuales se encuentra aquella que se denominó Promedio de Publicación Diaria definida según se indica en la Ecuación 1.

$$
\frac{\text { Count(Feed_Id) }}{(\text { Max(Fecha_Publicacion) }- \text { Min(Fecha_Publicacion) })}
$$

Donde se determina con claridad en el cuadro 3 que el número máximo de publicaciones diarias lo realiza la FanPage Universidad Central del Ecuador, con más de 15 posteos, y la que menos publica es Memes_PUCE, con un promedio diario de 0,12.

\begin{tabular}{lr}
\hline Nombres páginas & Promedio de publicación diaria \\
\hline Memes PUCE & 0,12 \\
Poli Memes & 5,19 \\
Memes UCE Quito & 0,34 \\
Universidad Central Del Ecuador & 15,56 \\
Escuela Politécnica Nacional & 4,83 \\
PUCE_Ecuador & 1,05 \\
\hline
\end{tabular}

Cuadro 3. Publicaciones diarias por Fanpage

Entre el contenido que publican las FanPages Oficiales, tienen un enfoque más informativo, siendo la publicación más relevante entre las tres, el vídeo realizado por PUCE_Ecuador, como se ve en la Figura 4 existe mucha diversidad de colores, incentivando a dejar una marca con el texto de: Celebrar cada logro obtenido, deleitarse con lo construido y dejar huella con cada paso, eso es ser de La Cato.

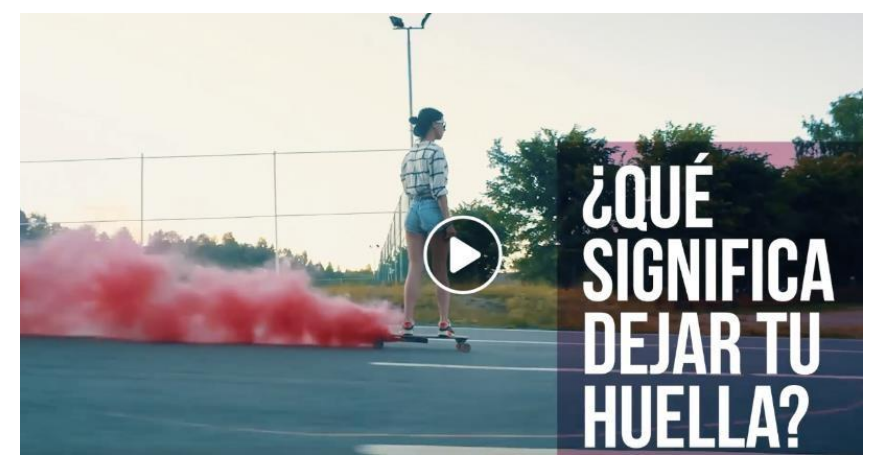

Figura 4. Mejor publicación didáctico en Facebook

Podemos aseverar que PUCE_Ecuador, es la FanPage didáctica más próxima a las páginas dedicadas al Ocio, donde en sus textos escribe frases más coloquiales, y con un enfoque de permitir conectarse a los estudiantes con la publicación.

\subsection{Tendencia en el tiempo}

\section{- Ocio en el tiempo}

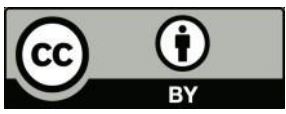


Gracias a la herramienta se ha estipulado cuáles son las tendencias e interacciones a través del tiempo que genera cada uno de sus Post, observando en la Figura 5 el comportamiento individual donde cada una posee su propio color.
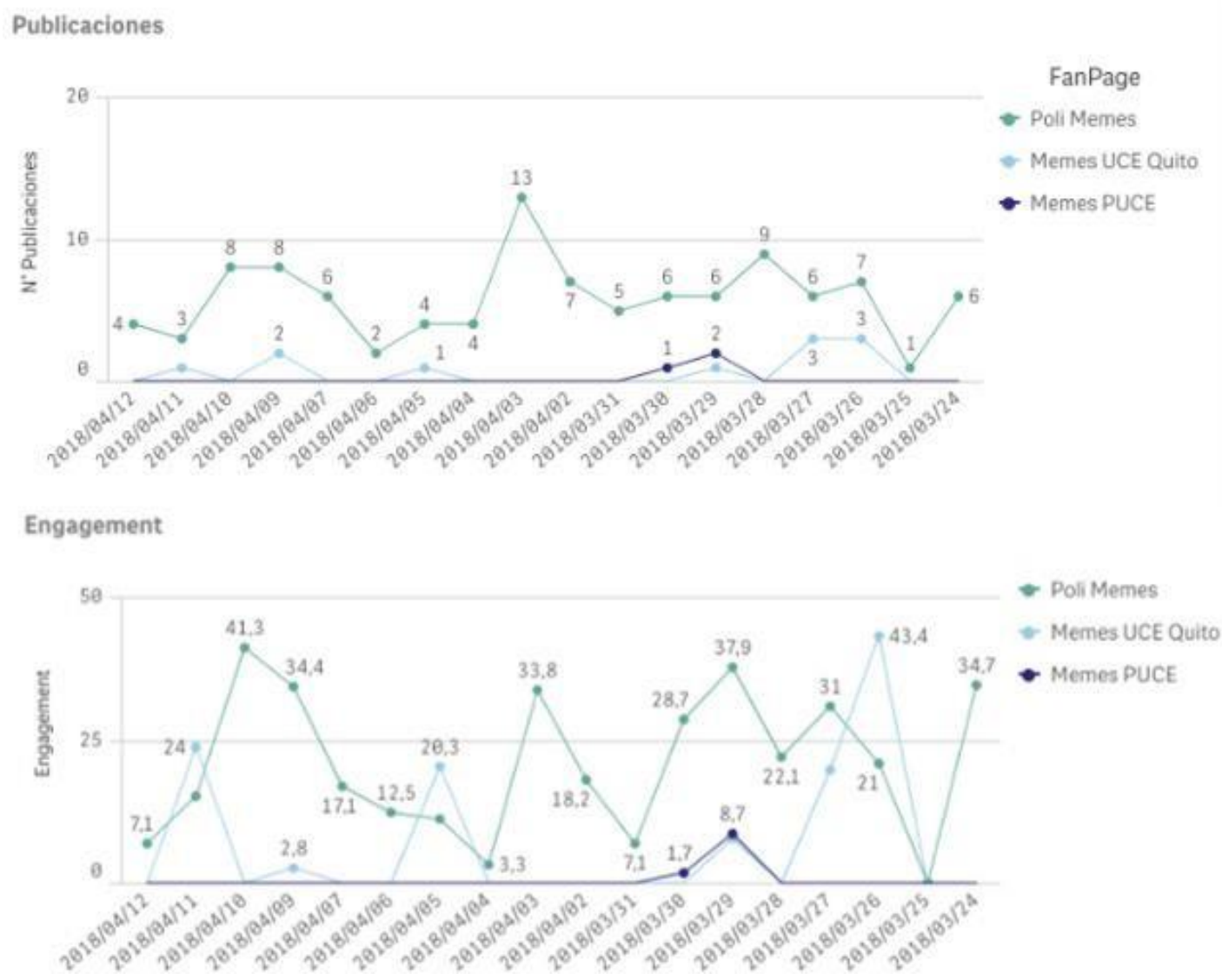

Figura 5. Ocio en el tiempo (abril-marzo)

Poli Memes es la FanPage que más publica en los días filtrados, generando un importante engagement en los estudiantes, con la observación de que no genera muchas comparticiones, limitándose a expandirse y viralizarse en la Red Social.

\section{- Académico en el tiempo}

A pesar de que las publicaciones en el ámbito Académico son mayores, superando las 7 publicaciones diarias en promedio, en la Figura 6 se observa que su índice de engagement es bajo, teniendo como pico más alto el 12,6 \% y en la comparación del engagement con respecto al Ocio en su mayoría de publicaciones superan el $20 \%$, teniendo casi el doble de interacciones con sus usuarios. Esta diferencia de casi 8 puntos refleja la inexactitud y poca creatividad de las páginas académicas a la hora de publicar su contenido, con ciertas excepciones que ya se han mencionado anteriormente. 


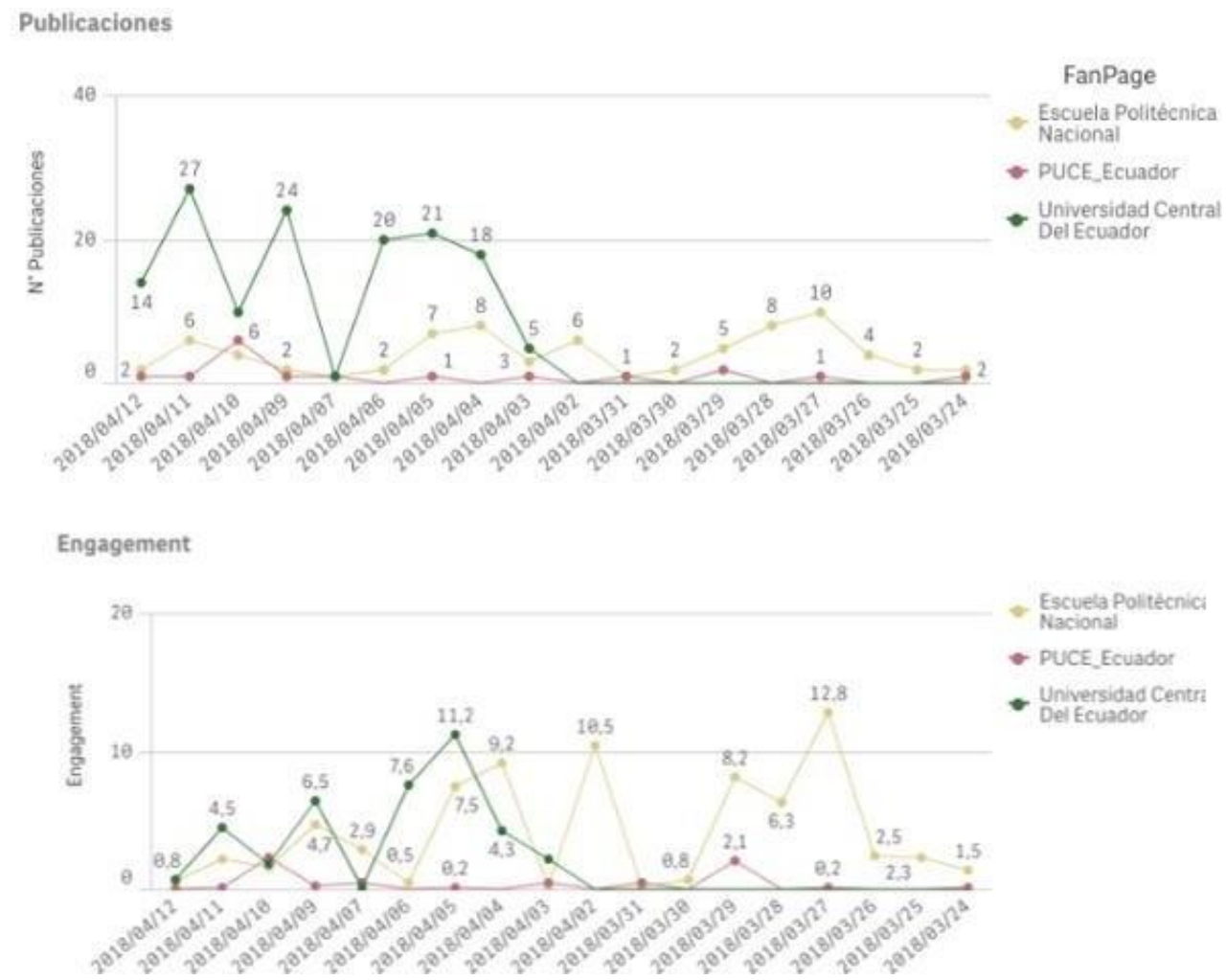

Figura 6. Académico en el tiempo (abril-marzo)

Una de las formas visuales que nos ha proporcionado la herramienta es el de observar todas las FanPages de forma conglomerada, para este análisis se han agrupado según las variables dependientes: ocio y académico. Se determinó que el día donde más publicaciones se han generado por parte de las FanPages de Ocio es el martes, una de las razones, es la atenuación de inicio de semana y la poca presión de las tareas finales para concluir los días laborables. Se observa en la Figura 7, que el día donde más se genera contenido de tipo Académico, es el miércoles, al examinar sus publicaciones, se evidencia la importancia de anunciar los eventos para días futuros a lo largo de las semanas o meses siguientes, la idea es considerar que el miércoles al ser un día que divide a la semana puede atraer más espectadores.

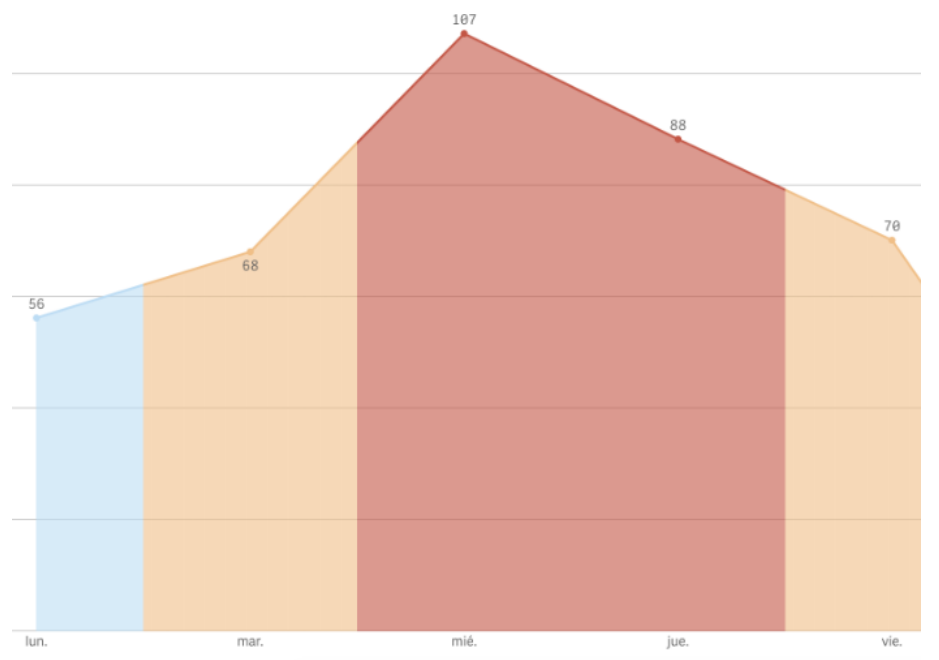

Figura 7. Día de más posteos académico 
- Estrategia de contenidos

Para alcanzar una determinada cifra de interacciones es necesario plantear una estrategia que considere un tipo de publicación específica, así como un día de la semana que favorezca e incremente el engagement en los seguidores o fans. La herramienta sugiere que para garantizar mayor número de compartidos se debe considerar publicar contenido de tipo video, los viernes, sin embargo, tras el desconocimiento de quienes administran estas FanPages, se ha determinado que se publica con mayor incidencia contenido de tipo foto, los miércoles, debilitando el número de interacciones esperadas.

- Detección de palabras

Uno de los aspectos claves para la generación de engagement es el uso apropiado del lenguaje y la lingüística, mismo que facilita el uso específico de palabras, en favor de cautivar a los seguidores y fans. Las palabras que suelen usarse son aquellas que se encuentran en tendencia y que de preferencia pueden ser usadas en clave o simbólicamente, aquellas que tienen un lenguaje sarcástico, o la fusión de un lenguaje coloquial y autóctono, así como palabras elegantes; la herramienta nos permite determinar aquellas palabras que más se han usado y que mayor número de interacciones han generado.

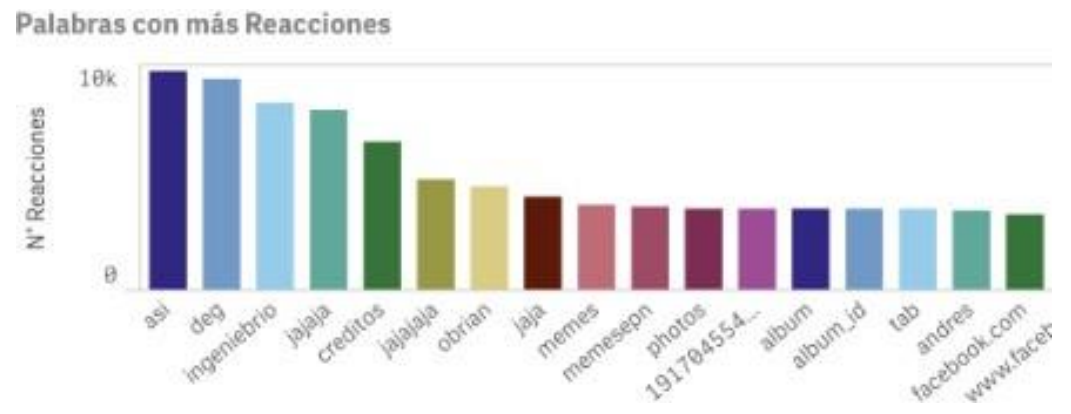

Figura 8. Palabras más usadas ocio

En la larga lista que se ha analizado, en la Figura 8 encontramos en orden jerárquico de mayor a menor impacto palabras como: memes, vos, combates, ingeniebrio, llave, etc., todas conllevan un mismo enfoque, dar la confianza a sus seguidores o fans para sentirse identificados, aceptados y que con la emoción del momento puedas convertirte en miembro de una gran comunidad digital.

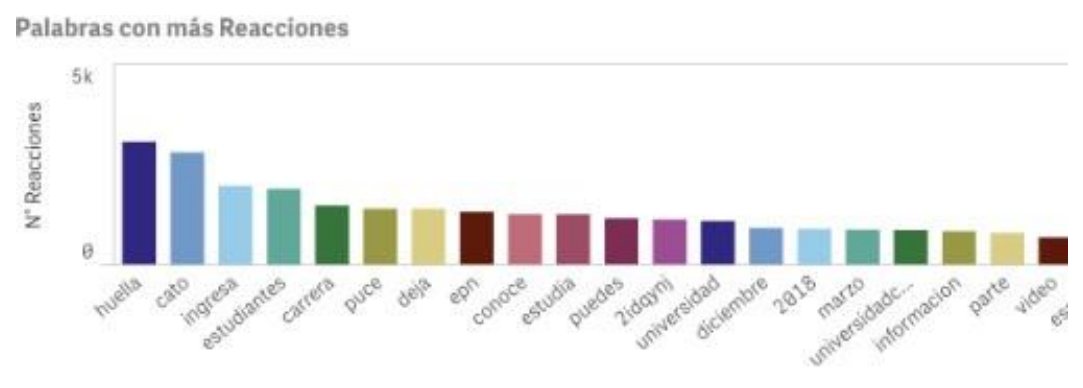

Figura 9. Palabras más usadas académico

Por otra parte, en las páginas destinadas a lo académico en la Figura 9, se ha encontrado palabras relevantes como: información, estudia, estudiantes, ingresa, participa, conoce, invitamos, carrera, ciencia, proceso, etc., donde su interés es claramente informativo, dejando de lado la creatividad, convirtiéndose en textos fríos llenos de palabras comunes. Cabe resaltar la labor que está haciendo PUCE_Ecuador, donde comienza a escribir palabras 
y frases más llamativas y coloquiales, como: la Puce, la Cato, sembrar, huella, la vida, sensación, único, que genera una emoción en los estudiantes, sobresaliendo frente a las demás FanPages Académicas, ya que apela a un sentido de apropiación e identidad muy necesaria en la vida universitaria.

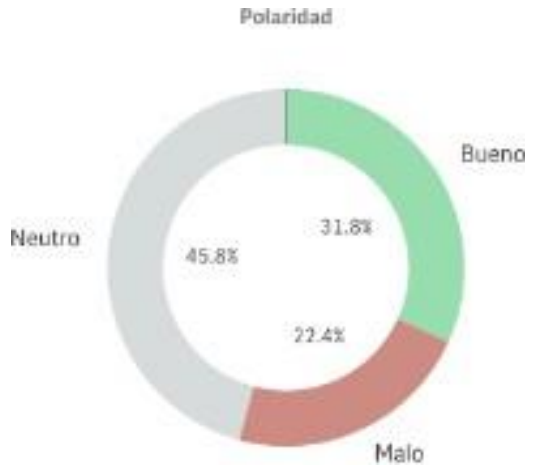

Figura 10. Análisis de sentimiento ocio

Cabe resaltar que el ocio genera más de 4000 comentarios, donde en su mayoría se hace énfasis en defender, resaltar a la universidad donde pertenecen y también a la vez desprestigiar y burlarse de las demás Universidades, como se ve en la Figura 10, existe un $22,4 \%$ de comentarios negativos donde se evidencia quejas y palabras groseras. Esta clase de comportamientos defensivos e incluso afectivos, marcan un acuerdo de conveniencia incluso entre seguidores y administradores para rentabilizar la atención de más seguidores o fans, a través de discusiones positivas y negativas en las redes.

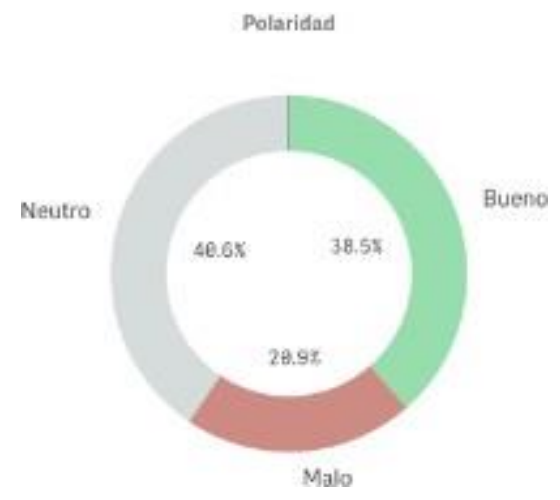

Figura 11. Análisis de sentimiento académico

Las FanPages Educativas generan un pequeño número de comentarios, como se ve en la Figura 11 en su mayoría son neutrales con un $40 \%$ solicitando más información en cursos, capacitaciones o eventos. La idea es dar a conocer la oferta académica de las universidades, y que se pueda consolidar un mayor número de participantes en las diferentes actividades.

\subsection{Propuesta de solución para un mejor impacto en el área académica}

\section{- Sustento para la propuesta}

Del análisis realizado a las publicaciones se puede observar que los universitarios tienden a responder de una manera más positiva a comentarios o frases que se manejen en un lenguaje coloquial sobre mensajes con un lenguaje formal habitual en las publicaciones de páginas universitarias. Los textos graciosos, son muy 
difundidos entre los universitarios, y cabe resaltar que incluso pueden contener información muy importante sumergida en ellos.

Mediante el análisis de contenido también se pudo determinar qué palabras claves son las que llaman la atención mucho más a los jóvenes, y cuáles son las que no se encuentran en su léxico. Además, se pudo determinar que los videos e imágenes son los contenidos que generaron más interacciones por parte de los estudiantes.

Del estado actual del conocimiento se puede extraer que uno de los aspectos muy importantes que generan sentimientos positivos en los usuarios es el poseer canales conversacionales con interacciones que no sea solamente unidireccionales, sino bidireccionales (Onieva, 2017).

Herramientas que han usado los chatterbots para el entorno educativo han generado respuestas muy positivas, como es el caso de la herramienta Agent socialMetric la cual logra distinguir las preguntas de los usuarios y luego elabora la respuesta adecuada a la oración recibida, creando líneas de colaboración y un excelente ambiente para el desarrollo de trabajos pedagógicos.

\section{- Planteamiento de la propuesta}

De acuerdo a los resultados obtenidos en el análisis y al marco teórico encontrado, se planteó un prototipo de agente conversacional virtual basado en inteligencia artificial. Como primer paso se necesitó diseñar una FanPage de Facebook para la Universidad Central del Ecuador, con el nombre UCE virtual didáctica en la cual se desplegó un chatbot desarrollado con la herramienta online Chatfuel, que brinda muchas facilidades al momento de crear chatbots, sobre todo para Facebook Messenger, pues cuenta con una secuencia de bloques para su construcción, a los cuales se les puede adherir videos, imágenes, audios, secuencias, e incluso plugins de correo electrónico.

Los aspectos que se tomaron en cuenta para el desarrollo del chatbot fueron el responder a preguntas comunes de los estudiantes universitarios y el brindar información acerca de eventos relevantes en la Universidad.

Este chatbot, permitirá obtener un canal conversacional de dos direcciones, entrenado en entender y responder a preguntas expresadas en un lenguaje coloquial, en el lenguaje que los jóvenes manejan. Además que, en su base de datos de preguntas y respuestas contará con las palabras más populares usadas en el léxico de los universitarios para poder interactuar con ellos de una manera más amigable y familiar para ellos. Otro aspecto de gran importancia es que el chatbot maneja contenido multimedia (videos, audios e imágenes) en su comunicación, generando de esta manera experiencias y sentimientos más marcados en los usuarios.

Este software servirá como un impulso para desarrollar en los estudiantes un sentimiento positivo hacia la página, creando así un ambiente universitario propicio para la comunicación entre profesores, alumnos y personal.

\section{Discusión}

Queda claro que las FanPages generan una gran aceptación por parte de los usuarios, y el grado de engagement depende de varios factores que se han analizado en esta investigación. 
Confirmando los resultados de estudios anteriores a este trabajo que muestran una carencia de habilidad por parte de las universidades a generar fidelidad en los estudiantes.

Todos estos resultados encontrados han servido como la base para la estructura de una posible solución, en busca de que el conocimiento sea impartido de una manera libre, sencilla, agradable y fácil para docentes y estudiantes.

Las FanPages de las tres universidades que analizamos tienen diferencias significativas en el número de sus seguidores, y de la misma manera nos muestran que el nivel de interacción no tiene una relación directa con el número de seguidores. Observando que las FanPages oficiales de las universidades a pesar de tener un significativo número de seguidores generan muy poca interacción. Posicionándose en los últimos lugares en la tabla de interacción en comparación a las FanPages de ocio, que con pocos seguidores genera mucha interacción.

Se ha comprobado en la investigación que el número de posteos diarios no es un factor determinante a la hora de obtener mayor cantidad de interacciones. De acuerdo con lo abordado en el marco teórico acerca del estudio de las universidades en redes sociales, contradiciendo a un conocimiento común y empírico que se tiene de que entre más publicaciones se realicen, existirá un mayor número de interacciones.

\subsection{Tópicos de interés para estudiantes en las redes sociales}

Es para todos claro que los jóvenes tienen un elevado conocimiento y uso de las redes sociales, siendo en la actualidad una preocupación debido a la gran influencia negativa en cuanto al rendimiento académico de los estudiantes, pero también se plantea un aspecto a favor el cual es que los mismos jóvenes presentan una gran aceptación y disposición para usar estas redes sociales para un fin académico. A demás podemos evidenciar que las palabras con mayor acogida son las que conllevan definiciones académicas fusionadas con un toque burlesco, siempre con un enfoque hacia el entretenimiento, lo novedoso y coloquial. Observamos en las FanPages oficiales de las tres Universidades su postura firme de regirse en un estado formal, en donde la mayoría de sus publicaciones son simples y fríos anuncios informativos que no generan ninguna experiencia atractiva en el usuario, de tal manera que se sienten poco identificados, sin la confianza y emoción para convertirse en un fan, mientras que en las tres FanPages de ocio, sus críticas hacia lo académico, social y lo que se encuentra en tendencia lo realizan de una manera jocosa, llena de contenido visual donde su fin es el entretenimiento llevando al estudiante a un estado mental que le permite sentirse identificado, reflejando muchas de sus perspectivas o anhelos en la pantalla.

\subsection{Las redes sociales en la educación}

En la actualidad las principales universidades del mundo han optado por estructurar una plataforma colaborativa en la que haya una contribución global compartiendo conocimientos entre los estudiantes y docentes donde también se imparten cursos, como en las plataformas Mooc's. En nuestros cálculos y resultados colocamos en el motor de búsqueda palabras como curso, deberes, libros, proyectos, etc. Todas ellas relacionadas con el tema de educación son muy pocas, observando que las FanPages oficiales se muestran reacias al uso de las redes sociales con fines educativos, limitándose a temas informativos y de poco interés.

\subsection{Respuesta a publicaciones}

Los comentarios que se realizan en las páginas de ocio son 4 veces más numerosos que los realizados en las páginas oficiales de las universidades. Según lo investigado y propuesto en la metodología se cree que esto es por la emoción de sentirse identificados, generando una 
polaridad en su mayoría positiva a diferencia de las oficiales, en las cuales se ve reflejada una posición neutra a sus publicaciones por parte de los estudiantes.

\subsection{Comportamientos de los estudiantes en las redes sociales}

Cuestiones como la identidad personal o la expresión del yo, se ponen en juego a la hora de que el usuario se defina en ser fan o seguidor, pues los universitarios, en la construcción de una imagen de aceptación, se dejan atraer por sus gustos y afinidades hasta elaborar incluso una propia máscara que les permita proyectarse para fines de diversión más que para asuntos académicos; por ello, las páginas oficiales de las instituciones de educación superior no se terminan de aproximar al reconocimiento de esta manera de interacción digital marcada por la población de los estudiantes universitarios.

Los comportamientos entonces serán variados, responderán a sus entornos, contextos, ideologías, apegos artísticos, pero sí con un denominador común, lo emocional. La singularidad de lo digital hace que el usuario construya su identidad a través de un personaje que puede seleccionar, controlar con el atenuante de la idealización de sí mismo, toda vez que no existe ese encontrarse con el otro cara a cara, de igual manera no responde forzosamente a lo simultáneo de la temporalidad que posee el mundo real o físico; por tanto, estos elementos lo distancian de la realidad a tal punto que se corre el riesgo de crear un universo virtual paralelo a lo que se llamaría espacio real.

\section{Conclusiones}

Se pudo observar que los estudiantes universitarios no llegan a sentir un verdadero compromiso con las páginas oficiales de Facebook de sus universidades, mientras que el engagement generado por sus pares dedicadas al ocio $\mathrm{y}$ entretenimiento, es considerablemente mayor.

Se logró determinar que el contenido audiovisual es un gran elemento a la hora de generar sentimientos y experiencias, aunque no reales, en la mente de las personas, forjando así un engagement sólido con las páginas que se valgan de estos recursos.

Sorprendentemente se observó que la cantidad de seguidores y de posteos de una FanPage, no es un factor determinante al momento de concebir interacciones por parte de los usuarios.

Por último, se encontró que las universidades han usado a las redes sociales como simples medios de publicación de boletines informativos, algo así como un tablero de hojas volantes, dejando así de aprovechar todo el potencial que ofrecen estos canales para captar el interés de los estudiantes. Por lo cual dependiendo del objetivo que se persiga, se podría pensar en un modelo hibrido que combine las dos tecnologías SQL y NoSQL, donde si se necesita mantener mayor consistencia se puede almacenar de una manera relacional mientras que para consultas inmediatas o recurrentes, se utilizarían bases de datos columnares.

\section{Recomendaciones y líneas futuras}

Se recomienda a las Universidades emplear elementos gráficos y de audio en los anuncios que deseen realizar a través de sus FanPages con el fin de generar experiencias más agradables y perdurables en los estudiantes. Se halla la necesidad de que en próximos estudios se analicen distintas redes sociales, como Twitter, YouTube, Instagram, etc. a fin de encontrar mayores características en los usuarios, dependiendo de la idiosincrasia propia de cada red. Se recomienda construir más canales de comunicación bidireccional entre las unidades educativas y los estudiantes, ya que se ha visto cómo pueden potenciar las relaciones entre ambos destinos.

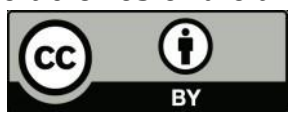

Licencia Creative Commons Atribución 4.0 Internacional (CC BY 4.0)

Revista Cátedra, 2(3), pp. 16-38, septiembre-diciembre 2019. e-ISSN: 2631-2875

https://doi.org/10.29166/catedra.v2i3.1713 


\section{Bibliografía}

Achen, R. (2016). Examining the Influence of Facebook Fans, Content, and Engagement on Business Outcomes in the National Basketball Association. Journal of Social Media for Organizations, 3(1), 1-15. Obtenido de http://www2.mitre.org/public/jsmo/pdfs/03-01-nba-business-outcomes.pdf

Anaza, N., \& Zhao, J. (2013). Encounter-based antecedents of e-customer citizenship behaviors. (E. G. Limited, Ed.) Journal of Services Marketing, 130-140. doi:http://dx.doi.org/10.1108/08876041311309252

Antolín Prieto, R. (2012). Youtube como paradigma del video y la telvisión en la web 2.0. Madrid: Universidad Complutense de Madrid. Obtenido de http://eprints.ucm.es/16111/

Brahimi, T., \& Sarirete, A. (2015). Learning outside the classroom through MOOCs. Computers in Human Behavior, 51, 604-609. doi:10.1016/j.chb.2015.03.013

Bustos, A., Flores, B., \& Flores, F. (2016). Las redes sociales, su influencia e incidencia en el rendimiento académico de los estudiantes de una entidad educativa ecuatoriana en las asignaturas de Física y Matemática. Latin-American Journal of Physics Education, 10(1), 1-7. Obtenido dehttps://dialnet.unirioja.es/servlet/oaiart?codigo=5517259

Calder, B., Malthouse, E., \& Schaedel, U. (2009). An experimental study of the relationship between online engagement and advertising effectiveness. Journal of Interactive Marketing, 23(4), 321-331. doi:10.1016/j.intmar.2009.07.002.

Ciechanowski, L., Przegalinska, A., Magnuski, M., \& Gloor, P. (2018). In the shades of the uncanny valley: An experimental study of human-chatbot interaction. Future Generation Computer Systems, 1-10. doi:10.1016/j.future.2018.01.055

Dakiche, N., Benbouzid-Si, F., Slimani, Y., \& Karima, B. (2018). Tracking community evolution in social networks: A survey,. Information Processing \& Management. doi:10.1016/j.ipm.2018.03.005.

Erat, P., Desouza, K., Schäfer-Jugel, A., \& Kurzawa, M. (2006). Business Customer Communities and Knowledge Sharing: Exploratory Study of Critical Issues. European Journal of Information Systems. (P. Macmillan, Ed.) European Journal of Information Systems, 15(5), 511-524. doi:10.1057/palgrave.ejis.3000643

Espuny, C., González, J., Mar, L., \& Gisbert, M. (2011). Actitudes y expectativas del uso educativo de las redes sociales en los alumnos universitarios. RUSC. Universities and Knowledge Society Journal, 171-185. Obtenido de http://www.redalyc.org/articulo.oa?id=78017126008

Gómez, M., Roses, S., \& Farias, P. (2012). El uso académico de las redes sociales en universitarios. Comunicar, 131-138. doi:10.3916/C38-2012-03-04

Gonzales, A. L., \& Hancock, J. (2010). Mirror, Mirror on My Facebook Wall: Effects of Exposure to Facebook on Self-Esteem. Cyberpsychology, Behavior, and Social Networking, 79-83. doi:10.1089/cyber.2009.0411

Gordon Salcedo, E. D., \& Noguera Vásconez, D. F. (2018). Estudio de los comportamientos y expectativas del uso de las rerdes sociales en las pincipales universidades del país y 
planteamiento de una posible solución para un mejor impacto en la actividad académica (Bachelor's thesis, Quito: UCE)

Herrera, D., Valerio, G., \& Rodríguez-Aceves, L. (2018). Digital engagement and social identity of sports fans: the case of Premier League teams on facebook. Revista Iberoamericana de Psicología del Ejercicio y el Deporte, 13(1), 59-69. Obtenido de http://www.redalyc.org/articulo.oa?id=311153534006

Herrera-Torres L., Pérez-Tur F., García-Fernández J., Fernández-Gavira J. (2017). El uso de las redes sociales y el engagement de los clubes de la Liga Endesa ACB. Cuadernos De Psicologia Del Deporte, 17(3), 175-182. Obtenido de http://www.redalyc.org/articulo.oa?id=227053840017

Huang, C. (2018). Social network site use and academic achievement: A meta-analysis. Computers \& Education, 119, 76-83. doi:10.1016/j.compedu.2017.12.010.

Junco, R. (2012). Too much face and not enough books: The relationship between multiple indices of Facebook use and academic performance. Computers in Human Behavior, 28(1), 187-198. doi:10.1016/j.chb.2011.08.026

Kim, J., Jin, B., \& Swinney, J. (2009). The role of etail quality, e-satisfaction and e-trust in online loyalty development process. Journal of Retailing and Consumer Services, 16(4), 239-247. doi:10.1016/j.jretconser.2008.11.019

Kudeshia, C., Mittal, A., \& Sikdar, P. (2016). Spreading love through fan page liking: A perspective on small scale entrepreneurs. Computers in Human Behavior, 54, 257 270. doi:10.1016/j.chb.2015.08.003

Kuz, A., Falco, M., Giandini, R., \& Nahuel, L. (2015). Integrando Redes Sociales y Técnicas de Inteligencia Artificial en Entornos Educativos. Revista Q, 10(19), 1-9. doi:10.18566/revistaq.v10n19.a04

Leskovec, J., Lang, K., Dasgupta, A., \& Mahoney, M. (2008). Statistical properties of community structure in large social and information networks. 695-704. doi:10.1145/1367497.1367591

Martelo, R., Jimenez-Pitre, I. 2., \& Martelo, P. (2017). Incidencia de las redes sociales en el rendimiento académico de los estudiantes de la universidad de La Guajira (Colombia). Revista Espacios, 38(45), $24 . \quad$ Obtenido de http://www.revistaespacios.com/a17v38n45/17384524.html

Onieva, J. E. (2014). El uso de las Redes Sociales en el ámbito de la comunicación universitaria andaluza. Revista Internacional de Relaciones Públicas, 4(8), 139-160. doi:10.5783/RIRP-8-2014-08-139-160

Onieva, J. E. (2017). Uso de las Redes Sociales por las universidades públicas andaluzas. (E. Complutense, Ed.) Estudios sobre el Mensaje Periodístico, 23(1), 631-645. doi:10.5209/ESMP.55618

Paniagua, F., Bernardo, G., \& Manuel, F. (2012). La incorporación de los departamentos de comunicación de las universidades españolas al entorno digital. Un análisis cuantitativo. Estudios sobre el mensaje periodístico, 18, 691-701. doi:10.5209/rev_ESMP.2012.v18.40948

Perez, R., Taheri, B., Farrington, T., \& O'Gorman, K. (2018). On being attractive, social and visually appealing in social media . Tourism Management $66,9$. 
Pöyry, E., Parvinen, P., \& Malmivaara, T. (2013). Can we get from liking to buying? Behavioral differences in hedonic and utilitarian Facebook usage. Electronic Commerce Research and Applications, 12(4), 224-235. doi:10.1016/j.elerap.2013.01.003

Rodríguez, A., \& Patricia, S. (2012). Análisis del uso de las redes sociales en Internet. ícono 14 Revista de comunicación y tecnologías emergentes, 10(2), 228-246. doi:10.7195/ri14.v10i2.198

Thakur, R. (2018). Customer engagement and online reviews. Journal of Retailing and Consumer Services, 41, 48-59. doi:10.1016/j.jretconser.2017.11.002

Wagner, C., \& Majchrzak, A. (2007). Enabling Customer-Centricity Using Wiki the Wiki Way. Journal of Management Information Systems, 23(3), 17-43. doi:10.2753/MIS07421222230302

Willet, L. H. (julio de 1974). A Model for Assessing Impact of Institutional Studies . ERIC, 11. Obtenido de A Model for Assessing Impact of Institutional Studies . 


\section{Autores}

SANTIAGO LEONARDO MORALES CARDOSO. Es doctor en informática por la Universidad de Alicante, España. Obtuvo el título de ingeniero informático, maestría en ciencias de la ingeniería y maestría en gestión informática empresarial en la Universidad Central del Ecuador.

Actualmente es docente en la Facultad de Ingeniería, Ciencias Físicas y Matemática.

MARIO RAÚL MORALES-MORALES. Es ingeniero de sistemas graduado en la Escuela Politécnica Nacional, Ecuador. Sus estudios de maestría en administración de negocios los realizó en la Universidad San Martín de Porres, Perú. Ha obtenido certificaciones en dirección de proyectos (PMI) y analítica de datos, con una extensa experiencia en proyectos empresariales en la región andina.

Actualmente es docente a la Facultad de Ingeniería, Ciencias Física y Matemática de la Universidad Central del Ecuador, y cursa el doctorado en informática en la Universidad de Alicante.

ESTEBAN GORDÓN-SALCEDO. Es ingeniero en informática graduado en la Universidad Central del Ecuador.

FRANZ NOGUERA-VÁSCONEZ. Es ingeniero en informática graduado en la Universidad Central del Ecuador.

Actualmente se desempeña como Gerente de Proyectos en una reconocida firma ecuatoriana de consultoría en analítica de datos. 